\title{
EL MARKETING EN EL DESARROLLO SOSTENIBLE DE LAS EMPRESAS FORESTALES: UN ACERCAMIENTO A LA CONCEPCIÓN CUBANA
}

Material original autorizado para su primera publicación en la revista académica REDMARKA. Revista Digital de Marketing Aplicado.

\footnotetext{
Yudemir Cruz Pérez

Ingeniero Forestal. Máster en Ciencias. Profesor Asistente de Economía Forestal. Departamento de Ingeniería Forestal. Universidad de Granma. Cuba. Carretera a Manzanillo, Km. 171/2, Peralejo, Bayamo. Granma. Cuba. Teléfono: (53)-(23)-48-10-15 Extensión: 187. Desarrolla actualmente su investigación doctoral en el tema: "Metodología para la elaboración de estrategias de marketing forestal sostenible en Cuba", en el Programa Internacional de Doctorado en Ciencias Forestales de la Universidad de Pinar del Río, Cuba. Acumula experiencia investigativa y docente en el área de la economía, administración y marketing forestal.
} 


\section{RESUMEN}

El objetivo de esta investigación fue elaborar una propuesta de conceptualización de las variables controlables del marketing en el marco del manejo forestal sostenible, para lo cual se realizó primero una investigación con fuentes secundarias que permitieran constatar la existencia de indicios que pudieran servir de base a la propuesta. Seguidamente se aplicó el proceso de solución de problema en grupo a través de un taller interinstitucional, de cuyo trabajo se obtuvieron los elementos fundamentales para conformar las nuevas concepciones. Como resultado se constató que el papel de un marketing mejorado en el sector forestal crecerá en importancia contribuir al desarrollo sostenible. Que el marketing en este nuevo contexto debe asumir la misión de reorganizar el comportamiento de la empresa, para crear una nueva forma de actuar en ellas y en todo el sistema de relaciones económicas y sociales. De igual modo los nuevos conceptos de las variables del marketing serán soporte de su puesta en práctica.

Palabras clave: marketing forestal, desarrollo sostenible, empresas forestales.

\section{ABSTRACT}

MARKETING IN THE SUSTAINABLE DEVELOPMENT OF FOREST ENTERPRISES: AN APPROACH TO THE DESIGN OF CUBAN

The objective of this research was to develop a proposal for a conceptualization of the controllable variables of marketing in the context of sustainable forest management, for which was first carried out research with secondary sources provide proof of the existence of evidence that could serve as a basis for proposal. The process was then applied problem solving in a group through inter-agency workshop, whose work has won the key elements to form new concepts. As a result it was found that the role of an improved marketing in forestry will become increasingly important contribution to sustainable development. That marketing in 
this new context must assume the task of reorganizing the conduct of the undertaking, to create a new way of acting on them and the entire system of economic and social relations. Likewise the new concepts of marketing variables will support its implementation.

Keywords: marketing forestry, sustainable development, forestry companies. 


\section{1.- ¿Por qué marketing forestal con enfoque sostenible?}

Actualmente la mayor parte del mundo está conciente de los problemas ambientales globales, por tanto, es posible conseguir al mismo tiempo la satisfacción de las necesidades de los consumidores, la maximización de los beneficios de la empresa y la minimización de los impactos negativos sobre el medio ambiente en la actividad de intercambio.

El marketing para la sostenibilidad, entonces, no busca comercializar productos ecológicos sacrificando las características necesarias para que este satisfaga las necesidades actuales del consumidor, sino que integra todos los subsistemas de la organización para lograr un fin más importante: la garantía de los recursos para el futuro.

En esencia, para conseguir el anhelado desarrollo sostenible, las empresas deben diseñar ofertas comerciales que permitan satisfacer las necesidades presentes de los consumidores, sin comprometer la capacidad de satisfacer las necesidades futuras de las próximas generaciones. De igual modo, el marketing para la sostenibilidad tiene una triple función: educar medioambientalmente a los consumidores y demás grupos de interés, rediseñar el marketing mix para incorporar el factor medioambiental y crear una cultura que trascienda las fronteras de la empresa.

El papel de un marketing mejorado en el sector forestal crecerá en importancia y permitirá a las empresas industriales y a las comunidades sacar ventaja de las oportunidades emergentes para contribuir al desarrollo sostenible. Es necesario entonces la adopción de nuevos conceptos para las variables controlables de marketing: producto, precio, promoción y distribución bajo los principios del manejo forestal sostenible en las condiciones de cada región y país.

Para las condiciones de Cuba la certificación forestal y el fortalecimiento de la cultura forestal son los puntos focales de las nuevas concepciones del Marketing para el Desarrollo Forestal Sostenible. 
Los recursos forestales, son considerados como una categoría histórico económica - social - natural por su propia esencia, inseparable de las conexiones entre la producción social y la naturaleza. Durante las últimas décadas la influencia de la humanidad sobre la naturaleza se ha ido incrementando en relación con la demanda existente de recursos naturales.

En el proceso productivo el hombre actúa como un elemento decisivo provocando cambios considerables en la naturaleza. La historia ha demostrado que el ser humano no racionaliza ni modera su utilización más que a partir del momento en que no puede satisfacer sus necesidades.

"El marketing como brazo visible de la empresa, se convierte en el centro de todas las críticas por cuanto su finalidad última es vender una mayor cantidad de producto. En definitiva, generar un mayor consumo. Hace muy pocos años, con la globalización de las consecuencias negativas derivadas de la tecnociencia, han aparecido en cantidades considerables los consumidores verdes". (Chamorro, 2006)

La aparición de este nuevo consumidor, preocupado no sólo por la satisfacción de sus necesidades actuales sino también por el futuro de la tierra, ha obligado a muchas empresas a adoptar nuevas estrategias de producción y naturalmente de comunicación. Esto es lo que ha dado origen al Marketing para el Desarrollo Forestal Sostenible (MDFS). 


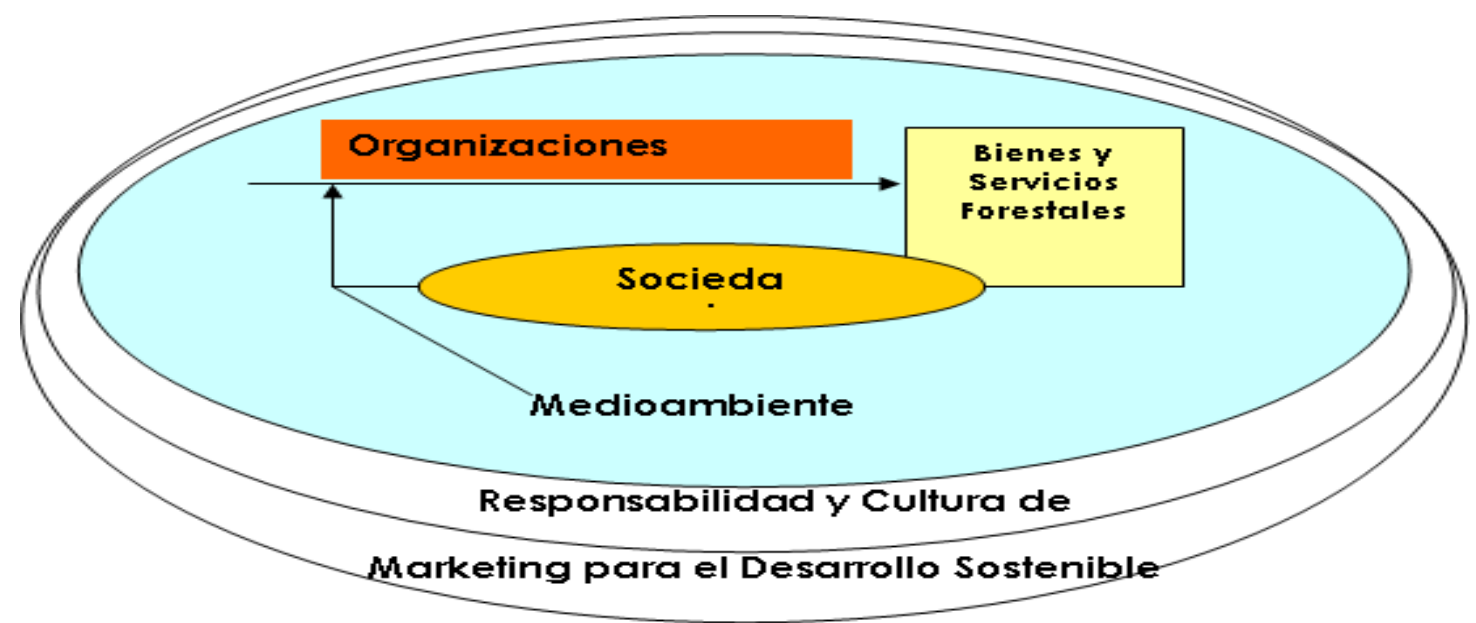

Figura. 1. Principio básico del marketing para el desarrollo sostenible.

"En la sociedad moderna, la producción y el consumo marchan por separado. El marketing los pone en contacto. Desde un punto de vista social, el marketing es una filosofía que muestra cómo crear sistemas eficaces de producción y, consiguientemente, cómo crear prosperidad. Los negocios son un subsistema de la sociedad, que cumple a la vez un papel económico y social. [Por lo tanto, una empresa debe operar de modo que haga posible producir beneficios para la sociedad y al mismo tiempo producir beneficios para la propia organización]. (Braidot, 1996)

El papel del MDFS en la sociedad implica grandes responsabilidades y retos. Además de la responsabilidad económica y social, es irremediable necesario poner énfasis en la responsabilidad medioambiental y la creación de una cultura de satisfacción de necesidades sustentablemente. Según algunas definiciones, la responsabilidad medioambiental forma parte de la responsabilidad social, un modo de ilustrarlas se presenta en el figura 1.

Tomando como referencia lo expuesto hasta aquí el objetivo de este trabajo fue elaborar una propuesta de conceptualización de las variables controlables del marketing en el marco del manejo forestal sostenible.

\section{2.- Aspectos metodológicos de la investigación.}


La investigación se desarrolló partiendo del análisis de la documentación existente sobre el desarrollo de la producción forestal en Cuba y las estructuras organizacionales que lo han sostenido en el tiempo. De igual modo el conjunto de documentos que en materia de marketing social fueron localizados.

El proceso lógico y cronológico de la investigación (figura 3), continuó con el desarrollo de un Taller Interinstucional en el cual se aplicó el Proceso de Solución de Problema en Grupos (PSPG); que según describen Arencibia y Sánchez (2005), instrumento que se estructura en seis pasos (figura 4), con la conformación de un comité de soluciones (integrado por todos los miembros participantes del taller), un facilitador encargado de guiar, organizar y encausar las actividades y discusiones, y un registrador, que llevó las memorias del taller y resumió lo expresado por todos.

En el taller participaron un total de 51 delegados que representaron 26 instituciones clave en el desarrollo forestal de Cuba. Se tomaron 25 intervenciones para generar las ideas iniciales, 45 ideas esenciales las cuales, luego de aclaradas, integradas y reducidas, fueron resumidas en 20 ideas. Éstas últimas permitieron formular los nuevos conceptos a los que se deseaban arribar. 


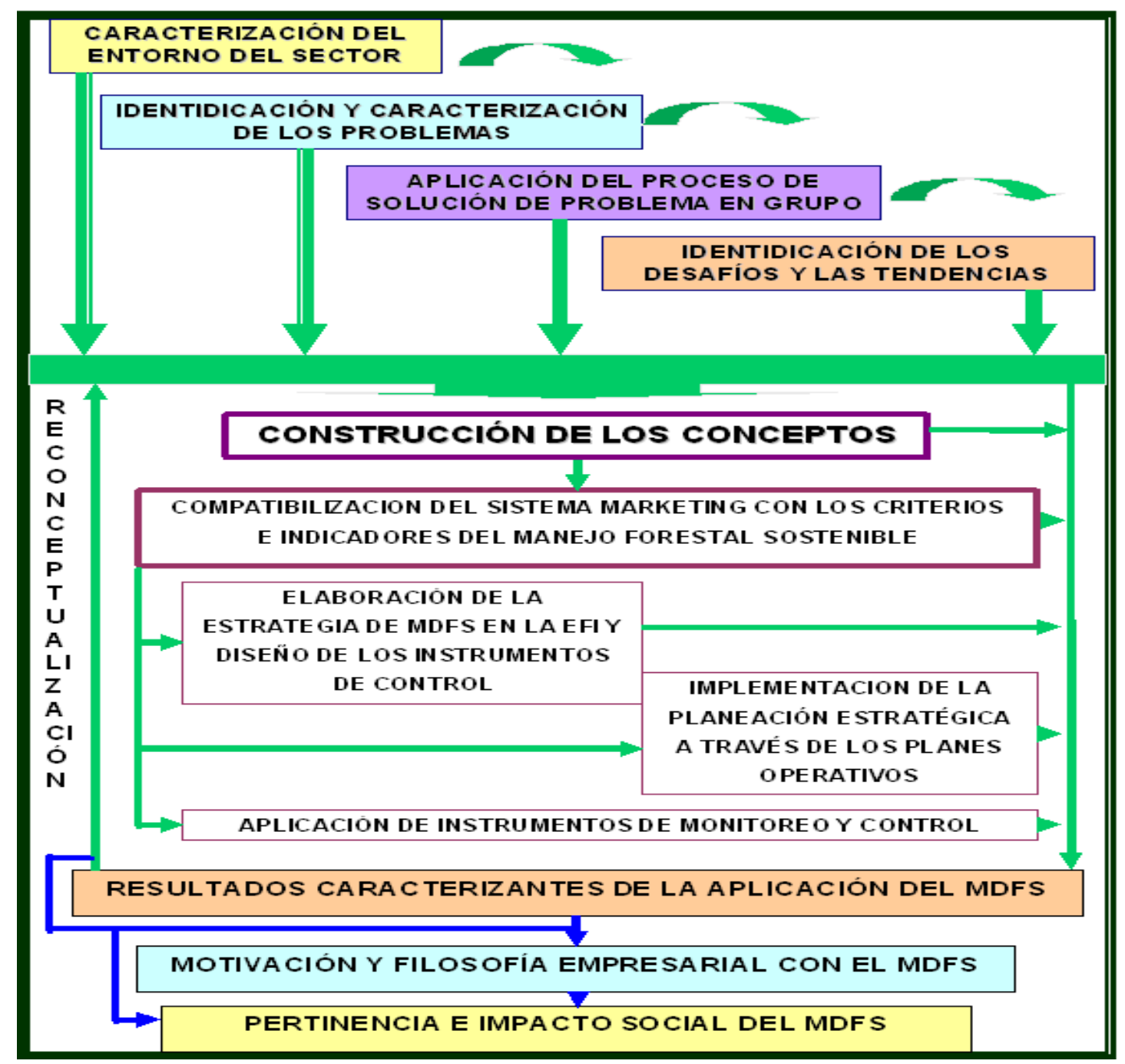

Figura. 3.- Proceso lógico de desarrollo de la filosofía empresarial del MDFS.

Fuente: Elaboración propia. 


\begin{tabular}{|c|c|c|c|c|}
\hline pasos & $\begin{array}{l}\text { preguntas a } \\
\text { responder }\end{array}$ & $\begin{array}{l}\text { Pxpansión } \\
\text { divergencia }\end{array}$ & $\begin{array}{l}\text { contracción } \\
\text { V } \\
\text { convergencia }\end{array}$ & $\begin{array}{l}\text { ¿Que es necessario } \\
\text { para pasar al passo } \\
\text { siguiente? }\end{array}$ \\
\hline $\begin{array}{l}\text { (1) } \\
\text { Identibicación y } \\
\text { selección de un } \\
\text { problema }\end{array}$ & $\begin{array}{l}\text { ¿Qué deseamos } \\
\text { Cambiar? }\end{array}$ & $\begin{array}{l}\text { Grupo } \\
\text { de problemas } \\
\text { a considerar }\end{array}$ & $\begin{array}{l}\text { Presentación de } \\
\text { un problema y una } \\
\text { "condición deseada" } \\
\text { convenida }\end{array}$ & $\begin{array}{l}\text { Identificación de la } \\
\text { "condición deseada" } \\
\text { en términos } \\
\text { obserrables }\end{array}$ \\
\hline$\underset{\text { Analisis }}{(2)}$ & $\begin{array}{l}\text { ¿Qué nos impide } \\
\text { alcanzar la } \\
\text { "coondición } \\
\text { deseada"? }\end{array}$ & $\begin{array}{l}\text { Grupo de causas } \\
\text { principales } \\
\text { identificadas }\end{array}$ & $\begin{array}{c}\text { Causa[s] clave[s] } \\
\text { identificada[s] } \\
\text { y verificada[s] }\end{array}$ & $\begin{array}{l}\text { Causa[s] clave[s] } \\
\text { comprobada[s] } \\
\text { y clasificada[s] }\end{array}$ \\
\hline $\begin{array}{l}\text { J } \\
\text { re } \\
\text { ones } \\
\text { iales }\end{array}$ & $\begin{array}{l}\text { ¿Cómo } \\
\text { podríamos } \\
\text { lograr un } \\
\text { cambio? }\end{array}$ & $\begin{array}{l}\text { ¿Cómo } \\
\text { podríamos } \\
\text { resolyer el } \\
\text { problema? }\end{array}$ & $\begin{array}{l}\text { Soluciones } \\
\text { potenciales } \\
\text { esclarecidas }\end{array}$ & $\begin{array}{l}\text { Lista de soluciones } \\
\text { potenciales }\end{array}$ \\
\hline $\begin{array}{l}\text { Seleccione } \\
\text { y planee la } \\
\text { solución del } \\
\text { problema }\end{array}$ & $\begin{array}{l}\text { ¿Cual es la } \\
\text { mejor manera } \\
\text { de hacerlo? }\end{array}$ & $\begin{array}{l}\text { Grupo decriterios } \\
\text { para evaluar las } \\
\text { soluciones poten- } \\
\text { ciales Grupo de } \\
\text { ideas sobre como } \\
\text { Anlicar yeraluar } \\
\text { la solucion } \\
\text { seleccionada }\end{array}$ & $\begin{array}{l}\text { Criterios para } \\
\text { evaluar las solucio - } \\
\text { nes convenidas } \\
\text { Planes de solución } \\
\text { y eraluación } \\
\text { convenidos }\end{array}$ & $\begin{array}{l}\text { Plan para ejecutar y } \\
\text { controlar el cambio } \\
\text { Criterio de medición } \\
\text { para evaluar la } \\
\text { eficacia de la } \\
\text { solución }\end{array}$ \\
\hline $\begin{array}{l}\text { (5) } \\
\text { Ponga en } \\
\text { práctica la } \\
\text { solución }\end{array}$ & $\begin{array}{l}\text { ¿Estamos } \\
\text { siguiendo el } \\
\text { plan? }\end{array}$ & & $\begin{array}{l}\text { Pupsta an práctica } \\
\text { [si fuera neces.ario] } \\
\text { de los planes de } \\
\text { contungencia } \\
\text { convenidos }\end{array}$ & $\begin{array}{l}\text { Solución } \\
\text { en su lugar }\end{array}$ \\
\hline $\begin{array}{l}\text { Evalue la } \\
\text { solución }\end{array}$ & $\begin{array}{l}\text { ¿Qué } \\
\text { resultados } \\
\text { dió? }\end{array}$ & & $\begin{array}{l}\text { Eficacia de la solu- } \\
\text { ción contenida. } \\
\text { Problemas identifica } \\
\text { dos [sı los hay] que } \\
\text { nersistan }\end{array}$ & $\begin{array}{l}\text { Verificación de que el } \\
\text { problema esta resuelto } \\
\text { y acuerdos para } \\
\text { enfocar problemas } \\
\text { que persistan }\end{array}$ \\
\hline
\end{tabular}

Figura. 4.- Proceso de solución de problema en grupo.

Fuente: Arencibia y Sánchez. 2005. p. 3.

\section{3.- Tratamiento del subsistema marketing en el sistema de producción} forestal.

\section{1.- Marco internacional.}

El subsistema marketing constituye un eje transversal en la proyección del sistema de producción forestal, entendiéndose, como una postura o filosofía adoptada por las organizaciones que desarrollan la gestión de este proceso productivo. 
La gestión forestal sostenible entonces, tiene como objeto asegurar que todos los bienes y servicios derivados del bosque satisfagan las necesidades de hoy a la vez que aseguran su disponibilidad y contribución continuadas a largo plazo.

"En su sentido amplio, abarca aspectos administrativos, jurídicos, técnicos, económicos, sociales y ambientales de la conservación y utilización de los recursos forestales. Implicando varios grados de intervención humana deliberada que comprende una variedad de acciones que van desde la salvaguarda y mantenimiento del ecosistema forestal y su función hasta favorecer especies o grupos de especies socialmente específicas o económicamente valiosas para la producción mejorada y perdurable de los bienes y servicios" (Overdevest y Rickenbach, 2010).

Un aspecto estrechamente relacionado con el marketing y el medio ambiente y al cual se le está prestando una gran atención es el de la certificación de los productos forestales, que pretende vincular el comercio internacional con la ordenación sostenible de los recursos forestales, alentando a los clientes a comprar únicamente productos fabricados con madera procedente de bosques ordenados de forma sostenible.

La certificación es el medio más concreto y efectivo para promover y asegurar el manejo sostenible de los recursos forestales y afines. Es un instrumento de marketing que garantiza y demuestra al consumidor que la madera u otro producto forestal, maderable o no, procede de un bosque gestionado de manera responsable y sostenible.

Permite enviar al comprador del producto un mensaje simple sobre su calidad y / o características. Se basa en: normas técnicas, prestigio, confianza y seriedad de quien hace la certificación (World Wildlife Fund / Adena, 2008).

Cada vez más consumidores, principalmente en Europa y Estados Unidos de América prefieren maderas o productos forestales provenientes de bosques 0 plantaciones, gestionadas de modo sustentable. Por esta razón es que han 
surgido innumerables sistemas de certificación de gestión forestal y etiquetado de productos forestales.

La figura dos, muestra como se distribuyen los 145, 5 millones de hectáreas de bosques certificados, a inicios del siglo XXI, entre los principales sistemas de certificación en el mundo.

Además de la certificación, el mundo forestal a nivel internacional para conseguir que la calidad del medio ambiente no se vea afectada negativamente por las decisiones de marketing en la intervención de los recursos forestales, es necesario incorporar objetivos ecológicos a cada una de las políticas de marketing.

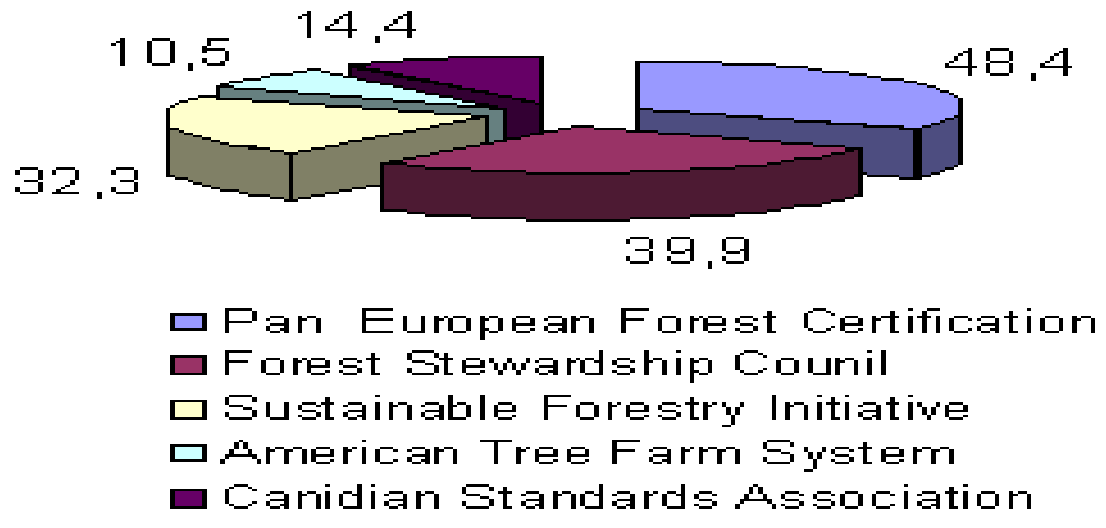

Figura. 2.- Superficie forestal certificada en el mundo (en millones de hectáreas).

Fuente: Elaboración propia, basado en FAO, 2009.

En este sentido, es necesario quedar claro que no se trata de enfrentar a los objetivos económicos de cada variable de marketing con los objetivos ecológicos. Se trata de buscar la consecución de ambos a la vez.

El marketing para el desarrollo sostenible no es solamente un conjunto de técnicas destinadas a diseñar y comercializar productos menos perjudiciales para el entorno natural; es, también, una forma de entender las relaciones de intercambio, 
basada en buscar la satisfacción de las tres partes que intervienen en ella: el consumidor, la empresa y el medio ambiente.

"Como sucede con la implantación de una filosofía marketing, aplicar la filosofía que hay detrás del marketing para el desarrollo sostenible no se puede limitar a implantar un departamento de esta área que aplique sus técnicas. Adoptar esta filosofía exige que el respeto por el medio ambiente impregne todo el comportamiento de la organización, no solamente los aspectos comerciales" (Chamorro, 2006).

Por tanto, esta nueva visión del marketing tiene la función de desarrollar a través de acciones internas una cultura empresarial basada en la preocupación medioambiental. Cultura que debe ser aceptada por todos los trabajadores de la organización, independientemente de su nivel jerárquico (desde la alta dirección hasta los operarios de línea) e independientemente de su función.

En definitiva, podemos considerar que en el mundo forestal el marketing no será ecológico y enmarcado en los principios de la sostenibilidad si no existe una gestión medioambiental de la empresa.

\section{2.- Tratamiento en Cuba.}

La certificación forestal, aspecto tratado en el epígrafe anterior, constituye un reto para el sector forestal de Cuba, pues aún no se cuenta con un sistema de certificación forestal propio.

"En tal sentido se han trazado objetivos, metas y actividades para iniciar la certificación de bosques en Cuba. Entre las metas se han definido la creación de un comité de iniciación para la certificación, la identificación y consulta de los tenentes de patrimonio, la difusión del tema y la formulación de una propuesta a las autoridades competentes para un pilotaje "(Herrero, 2004). 
Otro aspecto importante en Cuba para materializar la concepción de MDFS, lo constituye el capital humano especialmente entrenado en estos aspectos. Algunas de las necesidades de entrenamiento están relacionadas con el personal operativo, que deberá beneficiarse a corto plazo de los cursos de formación. Para satisfacer las necesidades a largo plazo, se debe desarrollar y fortalecer una formación de nivel universitario en el marketing de productos forestales.

\section{3.- El sector forestal cubano frente a los desafíos del marketing.}

La producción forestal, como actividad económica, depende del estado actual y de las posibilidades futuras de los recursos forestales del país. Por sus características, el manejo de los bosques requiere que cualquier intervención sea moderadamente pensada y planificada tanto en el espacio como en el tiempo.

Los efectos de un manejo inadecuado de los recursos forestales, se reflejarán durante un tiempo prolongado en el futuro y además se necesitarán una gran cantidad de años para enmendarlos.

"La producción total del sector forestal en Cuba a escala de la economía nacional no es de gran significación, esta peculiaridad no es sólo consecuencia de la disponibilidad de bosques en condiciones de ser explotados con fines económicos, sino también de la carencia de tecnologías modernas y el grado de obsolescencia de las existentes, que impiden realizar un aprovechamiento integral de las masa forestales" (González, 1996).

Las Empresas Forestales Integrales (EFI), constituyen el eslabón fundamental de la producción forestal dentro del complejo sistema de relaciones de la economía nacional, encargado de satisfacer las necesidades materiales y espirituales del ser humano pero a la vez buscando que su aparato productivo funcione con el máximo nivel de eficiencia. 
La situación actual de las EFI exige de un estudio profundo y analítico para buscar métodos científicamente fundamentados que permitan elevar la eficiencia económico - productiva.

El Proceso de Producción Forestal, visto como centro dentro del marco de las EFI, tiene como objetivo producir bienes y servicios forestales que satisfagan las necesidades de los clientes, y este presenta además características muy peculiares que lo diferencian de otros procesos, tales como:

- El carácter renovable de los recursos del bosque.

- La necesidad de grandes reservas maderables en pie.

- La larga duración del ciclo de producción.

- La variedad y diversidad de productos forestales que se obtienen del proceso de producción y de las funciones útiles del bosque, incluyendo valores directos e indirectos.

El sector forestal cubano refleja la necesidad de un fortalecimiento en su base organizacional, capaz de sostener Procesos como el Perfeccionamiento Empresarial (PPE), con estructuras planas y flexibles acorde con las tendencias actuales, y de un actuar con un enfoque de gestión de MDFS, trabajar en la puesta en práctica de los principios de marketing como sistema de pensamiento y acción para lograr la eficiencia en la gestión empresarial teniendo como núcleo al cliente y la responsabilidad social.

"Una vez que todo el tejido empresarial se inserte en el PPE se incrementará la posición de los productos en el mercado, debido a que el eje central del proceso es la gestión de calidad y de esta forma la satisfacción de las necesidades de los consumidores" (García, 2004).

\section{4.- Aquí está nuestro punto de partida en la concepción de MDFS.


Construir concepciones que puedan conducir al desarrollo del pensamiento universal en temas que escapan, en la mayoría de los casos, de las manos del grupo líder, puede considerarse paradigmático, más cuando la sostenibilidad sitúa al hombre como ente protagónico del mismo. Para el sector forestal cubano la concepción del MDFS descansa en los nuevos pilares de las variables controlables del marketing:

- Producto: Todo bien o servicio forestal con certificación de origen y calidad técnica, susceptible de ser ofrecido para satisfacer una necesidad o deseo de un cliente responsable.

- Precio: Es la cantidad de dinero que se paga por obtener un producto, así como el tiempo, esfuerzo, molestia, afectaciones medioambientales y sociales y la certificación de origen y calidad técnica, necesarias para obtenerlo.

- Promoción: Conjunto de actividades que tratan de comunicar los beneficios que reporta el producto y sus certificados de origen y calidad técnica, educando y persuadiendo a los consumidores en la responsabilidad de su uso racional al comprarlo a quien lo ofrece y en el apoyo al manejo forestal sostenible, buscando la identidad sociocultural y creando los valores perdurables de consumo responsable de los bienes y servicios forestales.

- Distribución: Conjunto de actividades que relaciona la producción forestal sostenible con el consumo, así como el conjunto de herramientas usadas para el monitoreo y control del destino del producto y su consumo responsable.

\section{CONSIDERACIONES FINALES}

El papel de un marketing mejorado en el sector forestal crecerá en importancia y permitirá a las empresas industriales y a las comunidades sacar ventajas de las oportunidades emergentes para contribuir al desarrollo sostenible. 
Para conseguir el anhelado desarrollo sostenible, las empresas deben diseñar ofertas comerciales que permitan satisfacer las necesidades presentes de los consumidores, sin comprometer la capacidad de satisfacer las necesidades futuras de las próximas generaciones. Para ello, el MDFS debe asumir como misión reorganizar el comportamiento de la empresa, con la finalidad de contribuir a superar la tremenda crisis medio ambiental que enfrenta el planeta.

En Cuba, el MDFS es aún una idea muy joven y ni siquiera en vías de desarrollo aún, pero sí constituye el punto de partida para una nueva forma de actuar de las EFI y todo el sistema de relaciones económicas y sociales.

Es necesario el cambio de postura de los líderes y una mayor apertura a la filosofía de marketing, tan deprimida durante las últimas décadas del siglo $\mathrm{XX}, \mathrm{y}$ que aparece recientemente por una necesidad de afrontar la sostenibilidad del manejo forestal, compatibilizando los intereses económicos con los medioambientales y socioculturales.

\section{BIBLIOGRAFÍA CITADA}

Arencibia, G. M.; Sánchez H. C (2005). Compendio sobre proceso de solución de problemas en grupo. Universidad de Granma. (Inédito).

Braidot, N. (1996). Marketing total. Argentina. 5ta edición ampliada. Macchi Grupo Editor S. A.

Chamorro, A. (21-10-2006). EL MARKETING ECOLÓGICO. [recuperado 21-102006] http://www.5campus.org/leccion/ecomarketing

FAO. (10-12-2009). LA PROMOCIÓN DE LA ORDENACIÓN SOSTENIBLE DE BOSQUES Y TERRENOS BOSCOSOS. [recuperado 10-12- 2009] de www.fao.org/forestry/site/13087/sp

García, Y. 2004. El papel del marketing en el tejido empresarial. Pinar del Río. Inédito. 
González, M. 1996. Los programas estratégicos de carácter sectorial. Experiencias en el sector de la resina en Cuba. Universidad de La Habana. Tesis (en opción al grado científico de Doctora en Ciencias Económicas). Ministerio de Educación Superior. Cuba.

Herrero, J. (2004). Estudio de tendencias y perspectivas del sector forestal en América Latina. Informe Nacional - Cuba. Dirección Forestal. MINAG.

Overdevest, Ch.; Rickenbach M. G.: «Forest certification and institutional governance: An empirical study of forest stewardship council certificate holders in the United States» Forest Policy and Economics 9, pp. 93- 102. 2006.

World Wildlife Fund I Adena. (17-5-2008). BASIC ELEMENTS OF GOOD PRACTICE AND SUSTAINABLE FOREST MANAGEMENT. [recuperado 17-5 2008] de http://www.cbp.org/07y31.html

Cuadro. 1.- Concepción / orientación de la relación de intercambio.

\begin{tabular}{|c|c|c|}
\hline Competencia & Orientación & Énfasis \\
\hline $\begin{array}{l}\text { Nula o mínima } \\
\text { (demanda mayor que } \\
\text { oferta) }\end{array}$ & Producción & $\begin{array}{l}\text { Producción y distribución. Lo importante es } \\
\text { disponibilidad del producto. Se parte del supuesto de } \\
\text { que todo lo que se produce se vende (Porque la } \\
\text { demanda supera la oferta). }\end{array}$ \\
\hline $\begin{array}{l}\text { Incremento (mayor } \\
\text { equilibrio entre } \\
\text { demanda y oferta) }\end{array}$ & Producto & $\begin{array}{l}\text { Calidad del producto. Se supone que si el producto } \\
\text { tiene calidad será demandado, sin necesidad de } \\
\text { promocionarlo (Pero la calidad sola no basta). }\end{array}$ \\
\hline $\begin{array}{l}\text { Fuerte (oferta mayor } \\
\text { que la demanda) }\end{array}$ & Ventas & $\begin{array}{l}\text { Promoción. Se trata de vender lo que se produce. Se } \\
\text { supone que los consumidores pueden ser inducidos a } \\
\text { comprar un producto, aun cuando no satisfaga una } \\
\text { necesidad. (Pero el cliente no satisfecho = cliente no } \\
\text { leal). }\end{array}$ \\
\hline $\begin{array}{l}\text { Fuerte (oferta mucho } \\
\text { mayor que la demanda) }\end{array}$ & Marketing & $\begin{array}{l}\text { Basado en el consumidor. Deben identificarse las } \\
\text { necesidades del consumidor y tratar de satisfacerlas, } \\
\text { obteniendo un beneficio. Deben considerarse también }\end{array}$ \\
\hline
\end{tabular}


la responsabilidad social de la entidad que ofrece sus productos o servicios.

Fuerte (crisis Desarrollo Basado en los indicadores de la sostenibilidad. No estructurales, con Sostenible basta ser responsables y pertinentes con la sociedad, énfasis en los impactos es manejar todos los bienes y servicios acordes a las medioambientales) exigencias de los clientes, generando solvencia para la empresa y sobre todo garantizando la satisfacción de las necesidades de las próximas generaciones.

Fuente: Adaptado y transformado de Santesmases, 1999.

Fuente: Basado en García, 2004.

\section{Para citar este artículo:}

Yudemir Cruz Pérez (29-04-2010). EL MARKETING EN EL DESARROLLO SOSTENIBLE DE LAS EMPRESAS FORESTALES: UN ACERCAMIENTO A LA CONCEPCIÓN CUBANA.

REDMARKA - CIECID - Unidad de Investigación en Marketing Aplicado-Universidad de A Coruña, Número 4, V1, pp.3-20

ISSN $1852-2300$

URL del Documento : http://www.cienciared.com.ar/ra/doc.php?n=1254

URL de la Revista : http://www.cienciared.com.ar/ra/revista.php?wid=39 